\title{
Which algorithm diagnoses invasive pulmonary aspergillosis best in ICU patients with COPD?
}

\author{
To the Editor:
}

Invasive pulmonary aspergillosis (IPA) is a potentially lethal opportunistic infection, mainly affecting immunocompromised patients, particularly those with prolonged neutropenia [1]. Several reports have shown that Aspergillus spp. can also cause IPA in patients with a priori less severe immune dysfunction, such as those in intensive care units (ICUs) [2-5] or with chronic obstructive pulmonary disease (COPD) [5-8]. In these patients, diagnosis of IPA remains a challenge, because the reference diagnostic criteria (defined by the European Organization for Research and Treatment of Cancer/Mycosis Study Group (EORTC/MSG)) were developed for research in high-risk patients and not specifically for patients in the ICU or patients with COPD [9]. Two alternative algorithms have been proposed for this setting: the COPD algorithm for patients with COPD [6] and the Clinical algorithm for patients in the ICU [10].

We compared the three algorithms to evaluate which of them is the most accurate for classifying critically ill patients with COPD and Aspergillus-positive cultures.

This study was part of the AspICU project [11], focusing on patients with COPD and Aspergillus-positive lower airway cultures. Collected data included demographics, clinical features, cultures, radiological findings (suggestive of IPA) and Global Initiative for Chronic Obstructive Lung Disease (GOLD) stage [12].

Using the COPD [6], Clinical [10] and EORTC/MSG [9] algorithms, patients were classified as having proven IPA, putative/probable IPA or colonisation. None of the patients could be classified as having "possible" IPA (as defined by the COPD and EORTC/MSG algorithms) because this requires negative cultures and all the patients in AspICU had at least one positive culture.

The validity of the algorithms was assessed against histological results when available. Algorithm sensitivities and specificities were compared using McNemar's Chi-squared test with continuity correction. The validity of diagnostic signs from the Clinical algorithm was also assessed; both putative and proven diagnoses were considered as IPA for this analysis. The Chi-squared test was used to rule out the hypothesis that diagnostic signs and Clinical algorithm results were independent. Statistical analyses were performed using R software (version 3.1.2; R Core Team, Vienna, Austria), and $\mathrm{p}<0.05$ was considered significant.

The AspICU study included 563 patients in the ICU [11], of whom 174 (31\%) had COPD. To obtain a more homogenous cohort, 32 patients were not included in this analysis (no lung involvement $(n=5)$; insufficiently documented COPD diagnosis $(n=6)$; neutrophil count $<500$ per $\mu \mathrm{L}(\mathrm{n}=2)$; solid organ transplantation $(n=12)$; immunosuppressive medication $(n=9)$; chemotherapy $(n=3)$; leukaemia $(n=3)$ and myelodysplastic syndrome $(n=3)$ ), leaving a total of 142 patients. The patient classifications according to the three algorithms are shown in table 1.

Using the COPD, Clinical and EORTC/MSG algorithms, mechanical ventilation was found to be required in, respectively, 26 out of 50, 39 out of 71 and 17 out of 25 patients with proven or probable/putative IPA compared with 1 out of 53, 6 out of 71 and 28 out of 117 patients, respectively, with colonisation.

Histological results were available for 23 patients: 13 had IPA and 10 had colonisation. In eight patients, IPA was diagnosed only after death. Before the autopsy results, four patients were classified as having 
TABLE 1 Final diagnosis and validity values using the three algorithms

\begin{tabular}{|c|c|c|c|c|c|c|c|c|c|}
\hline Diagnosis & \multicolumn{5}{|c|}{ GOLD stage } & \multicolumn{4}{|c|}{ All COPD cases } \\
\hline COPD algorithm & & & & & & $54 \%$ & $70 \%$ & $70 \%$ & $54 \%$ \\
\hline Probable (37) & 0 & 0 & 11 & 26 & 0 & & & & \\
\hline Colonisation (53) & 2 & 15 & 7 & 10 & 19 & & & & \\
\hline Inconclusive (39) & 0 & 20 & 3 & 5 & 11 & & & & \\
\hline Putative (58) & 0 & 15 & 8 & 26 & 9 & & & & \\
\hline Colonisation (71) & 2 & 20 & 13 & 15 & 21 & & & & \\
\hline EORTC/MSG algorithm & & & & & & $23 \%$ & $80 \%$ & $60 \%$ & $44 \%$ \\
\hline Proven (13) & 1 & 3 & 2 & 6 & 1 & & & & \\
\hline Probable (12) & 0 & 2 & 0 & 10 & 0 & & & & \\
\hline Colonisation (117) & 2 & 33 & 21 & 31 & 30 & & & & \\
\hline
\end{tabular}

The numbers in parentheses are the total number of patients in each category. COPD: chronic obstructive pulmonary disease; GOLD: Global Initiative for Chronic Obstructive Lung Disease; EORTC/MSG algorithm: European Organization for the Research and Treatment of Cancer/ Mycosis Study Group; PPV: positive predictive value; NPV: negative predictive value.

probable IPA and four as inconclusive using the COPD algorithm, while seven were classified as having putative IPA and one as having colonisation using the Clinical algorithm, and all eight were classified as having colonisation using the EORTC/MSG algorithm. Sensitivity, specificity and predictive values for the three algorithms are shown in table 1 (inconclusive diagnoses were classified as colonisation for these calculations). Overall, the diagnosis was correct (true positive or true negative) in 19, 12 and three cases using the Clinical, COPD and EORTC/MSG algorithms, respectively. The Clinical algorithm was more sensitive than the EORTC algorithm $(\mathrm{p}=0.013)$, while the sensitivity of the COPD algorithm was not significantly different from those of the other algorithms $(\mathrm{p}=0.13)$. The specificities of the three algorithms were not statistically different $(\mathrm{p}=1)$.

We also analysed the validity values of the clinical and radiological criteria from the Clinical algorithm to diagnose proven/putative IPA. Mechanical ventilation $(\mathrm{p}=0.0001)$ and nodules on chest radiograph ( $p=0.0364$ ) were most predictive of IPA, while absence of abnormalities on chest radiograph essentially excluded a diagnosis of IPA ( $p=0.0001)$. IPA-suggestive abnormalities on chest computed tomography scan were not significantly predictive of a diagnosis of IPA.

Before the histological results, GOLD II patients who fulfilled the criteria for probable IPA could not be classified using the COPD algorithm; this was the case for seven patients, of whom two had IPA confirmed histologically. Using the Clinical and EORTC/MSG algorithms, these cases would have been classified as putative (six out of seven) or probable (two out of seven), respectively. 16 other GOLD II patients were classified as inconclusive because several criteria were missing and none was receiving steroids (autopsy-confirmed IPA in one patient). Using the Clinical algorithm, seven of these 16 cases would have been classified as having putative IPA, while all would have been classified as having colonisation using the EORTC/MSG algorithm.

Patients with COPD are at risk of developing IPA [5-8]. However, use of biopsy to confirm diagnosis remains challenging in patients who are mechanically ventilated $[13,14]$. For patients in the ICU with COPD and Aspergillus-positive respiratory tract cultures, we compared three algorithms that have been proposed to discriminate colonisation from IPA. Comparisons in the 23 patients with histological data showed that a correct diagnosis was more often obtained using the Clinical algorithm, followed by the COPD algorithm and finally the EORTC/MSG algorithm. Interestingly, even GOLD II patients treated with steroids are at risk of developing IPA.

One of the algorithms is specific for patients with COPD [6] whereas the others $[9,10]$ are not. Moreover, the EORTC/MSG algorithm [9] has been validated only in immunocompromised patients, and patients in the ICU with COPD rarely meet the EORTC/MSG host factor criteria [9, 15]. Recently, BLOT et al. [11] reported that more cases of IPA in patients in the ICU were diagnosed using the Clinical algorithm than using the EORTC/MSG criteria, primarily because host factors were often not present in these patients. Our data confirm that patients are more often classified as having proven/putative IPA by the Clinical 
than by the EORTC/MSG algorithm. With both the COPD and EORTC/MSG algorithms, some patients could not be classified; for the former because colonisation criteria were not met or they were not GOLD III or IV (leading to 39 patients considered as inconclusive), and for the latter because COPD is not recognised as a host risk factor (leading to 116 patients being considered as having colonisation).

Among the 38 GOLD II patients, 15 were classified as having colonisation using the COPD algorithm, and 23 had inconclusive results. Among these patients, seven had the same features, clinical signs, mortality rate and radiological findings as the proven/probable IPA group; interestingly, two of these patients were shown histologically to have IPA.

Our study limitations include the absence of a strict procedure to diagnose IPA and the limited number of histological specimens. However, the patients were carefully reviewed, the study was performed in 30 centres from eight countries, and the Clinical algorithm has been validated recently [11]. Moreover, inclusion of histopathology-controlled cases allowed comparisons before and after histopathological results; for example, concordance was $89 \%$ using the COPD algorithm (data not shown). Another limitation is that our conclusions are only valid for patients with Aspergillus-positive cultures.

For patients in the ICU with COPD and Aspergillus-positive culture, the Clinical algorithm is more appropriate than the EORTC/MSG algorithm to discriminate IPA from colonisation. The COPD algorithm is less useful because of the numbers of inconclusive cases. IPA should be considered in patients with COPD, especially in those receiving systemic steroid therapy and who have a positive Aspergillus culture, respiratory failure and an abnormal chest radiograph, regardless of GOLD stage.

Pierre Bulpa ${ }^{1}$, Benoît Bihin ${ }^{2}$, George Dimopoulos ${ }^{3}$, Fabio S. Taccone ${ }^{4}$, Anne-Marie Van den Abeele ${ }^{5}$, Benoît Misset $^{6}$, Wouter Meersseman ${ }^{7}$, Herbert Spapen $^{8}$, Teresa Cardoso ${ }^{9}$, Pierre-Emmanuel Charles ${ }^{10}$, Jordi Rello $^{11}$, Dirk Vogelaers ${ }^{12}$ and Stijn Blot ${ }^{12}$ on behalf of the AspICU study investigators ${ }^{13}$

${ }^{1}$ Intensive Care Unit, CHU UCL Namur, Université Catholique de Louvain, Yvoir, Belgium. ${ }^{2}$ Scientific Support Unit, CHU UCL Namur, Université Catholique de Louvain, Yvoir, Belgium. ${ }^{3}$ Dept of Critical Care Medicine, Attikon University Hospital, University of Athens Medical School, Athens, Greece. ${ }^{4}$ Dept of Intensive Care, Erasme Hospital, Université Libre de Bruxelles, Brussels, Belgium. ${ }^{5}$ Dept of Microbiology, General Hospital St. Lucas, Ghent, Belgium. ${ }^{6}$ Medical Intensive Care Unit, University Hospital Charles Nicolle, Rouen Normandie University, Rouen, France. ${ }^{7}$ Dept of Internal Medicine, University Hospital Leuven, Leuven, Belgium. ${ }^{8}$ Intensive Care Dept, University Hospital, Vrije Universiteit, Brussels, Belgium. ${ }^{9}$ Dept of Intensive Care, Hospital de Santo Antonio, Porto, Portugal. ${ }^{10}$ Service de Réanimation Médicale, Hôpital Bocage Central, C.H.U. Dijon, Dijon University Hospital, Dijon, France. ${ }^{11}$ Hospital Universitari Vall d'Hebron, Vall D'Hebron, Institute of Research, CIBERES, Universitat Autonoma de Barcelona, Barcelona, Spain. ${ }^{12}$ Dept of Internal Medicine, Faculty of Medicine and Health Science, Ghent University, Ghent, Belgium. ${ }^{13} \mathrm{~A}$ full list of the AspICU study investigators and their affiliations can be found in the Acknowledgements section.

Correspondence: Pierre Bulpa, Intensive Care Unit, CHU UCL Namur, Université Catholique de Louvain, 5530 Yvoir, Belgium. E-mail: pierre.bulpa@uclouvain.be

Received: Sept 202016 | Accepted after revision: June 222017

Support statement: This study received an unrestricted educational grant from Pfizer Belgium and a research grant from Ghent University. S. Blot holds a research mandate from Ghent University. The AspICU project was endorsed by the European Critical Care Research Network of the European Society of Intensive Care Medicine. The funding bodies had no role in collecting, analysing or interpreting the data, and were not involved in the writing or approval of the final manuscript. Funding information for this article has been deposited with the Crossref Funder Registry.

Conflict of interest: Disclosures can be found alongside this article at erj.ersjournals.com

Acknowledgements: The AspICU Study Investigators are as follows: Miguel Blasco-Navalpotro, Hospital Universitario Severo Ochoa (Madrid, Spain); Stijn Blot, Ghent University (Ghent, Belgium); Nele Brusselaers, Ghent University Hospital, (Ghent, Belgium); Pierre Bulpa, Mont-Godinne University Hospital (Yvoir, Belgium); Teresa Cardoso, Hospital de Santo Antonio (Porto, Portugal); Pierre-Emmanuel Charles, Dijon University Hospital (Dijon, France); Didier Clause, Cliniques de l'Europe (Brussels, Belgium); Patricia Courouble, Cliniques Universitaires Saint Luc (Brussels, Belgium); Emmanuel De Laere, Heilig Hartziekenhuis Roeselaere-Menen (Roeselaere, Belgium); George Dimopoulos, University Hospital Attikon (Athens, Greece); Frédéric Forêt, Centre Hospitalier Régional Mons-Warquignies (Mons, Belgium); Dan Li, Shangai Public Health Clinical Center (Shangai, China); Claude Martin, Assistance publique hôpitaux de Marseille, (Marseille, France); Shahram Mashayekhi, Centre Hospitalier Grand Hornu (Hornu, Belgium); Wouter Meersseman, Universitair Ziekenhuis Gasthuisberg (Leuven, Belgium); Benoit Misset, Hôpital Saint-Joseph (Paris, France); José Artur Paiva, Hospital de Sao Joao, (Porto, Portugal); Paulo Mergulhao, Hospital de Sao Joao (Porto, Portugal); Alessandro Pasqualotto, Santa Casa-Complexo Hospitalar (Porto Allegre, Brazil); Marcos Pérez, Vall d'Hebron University Hospital (Barcelona, Spain); Ratna Rao, Apollo Hospital (Hyderabad, India); Jordi Rello, Joan XXIII University Hospital (Tarragona, Spain) and Vall d'Hebron University Hospital (Barcelona, Spain); Jessica Souto, Vall d'Hebron University Hospital (Barcelona, Spain); Herbert Spapen, Brussels University Hospital (Brussels, Belgium); Fabio Silvio Taccone, Hôpital Erasme (Brussels, Belgium); Anne-Marie Van den Abeele, AZ Sint Lucas, (Ghent, Belgium); Koenraad Vandewoude, Ghent University Hospital, (Ghent, Belgium); and Dirk Vogelaers, Ghent University Hospital (Ghent, Belgium). 


\section{References}

1 Meersseman W, Van Wijngaerden E. Invasive aspergillosis in the ICU: an emerging disease. Intensive Care Med 2007; 33: 1679-1681.

2 Lugosi M, Alberti C, Zahar JR, et al. Aspergillus in the lower respiratory tract of immunocompetent critically ill patients. J Infect 2014; 69: 284-292.

3 Vandewoude K, Blot S, Benoit D, et al. Invasive aspergillosis in critically ill patients: analysis of risk factors for acquisition and mortality. Acta Clin Belg 2004; 59: 251-257.

4 Taccone FS, Van den Abeele AM, Bulpa P, et al. Epidemiology of invasive aspergillosis in critically ill patients: clinical presentation, underlying conditions, and outcomes. Crit Care 2015; 19: 7.

5 Kosmidis C, Denning DW. The clinical spectrum of pulmonary aspergillosis. Thorax 2015; 70: $270-277$.

6 Bulpa P, Dive A, Sibille Y. Invasive pulmonary aspergillosis in patients with chronic obstructive pulmonary disease. Eur Respir J 2007; 30: 782-800.

7 Bulpa PA, Dive AM, Garrino MG, et al. Chronic obstructive pulmonary disease patients with invasive pulmonary aspergillosis: benefits of intensive care? Intensive Care Med 2001; 27: 59-67.

8 Delsuc C, Cottereau A, Frealle E, et al. Putative invasive pulmonary aspergillosis in critically ill patients with chronic obstructive pulmonary disease: a matched cohort study. Crit Care 2015; 19: 421.

9 De Pauw B, Walsh TJ, Donnelly JP, et al. Revised definitions of invasive fungal disease from the European Organization for Research and Treatment of Cancer/Invasive Fungal Infections Cooperative Group and the National Institute of Allergy and Infectious Diseases Mycoses Study Group (EORTC/MSG) Consensus Group. Clin Infect Dis 2008; 46: 1813-1821.

10 Vandewoude KH, Blot SI, Depuydt P, et al. Clinical relevance of Aspergillus isolation from respiratory tract samples in critically ill patients. Crit Care 2006; 10: R31.

11 Blot SI, Taccone FS, Van den Abeele AM, et al. A clinical algorithm to diagnose invasive pulmonary aspergillosis in critically ill patients. Am J Respir Crit Care Med 2012; 186: 56-64.

12 Global Initiative for Chronic Obstructive Lung Disease. Global strategy for the diagnosis, management and prevention of chronic obstructive pulmonary disease. NHLBI/WHO workshop report. Bethesda, National Heart, Lung and Blood Institute, 2001. Update of the Management Sections. www.goldcopd.com. Date last updated: 2016

13 Koulenti D, Garnacho-Montero J, Blot S. Approach to invasive pulmonary aspergillosis in critically ill patients. Curr Opin Infect Dis 2014; 27: 174-183

14 Koulenti D, Vogelaers D, Blot S. What's new in invasive pulmonary aspergillosis in the critically ill? Intensive Care Med 2014; 40: 723-726.

15 Meersseman W, Vandecasteele SJ, Wilmer A, et al. Invasive aspergillosis in critically ill patients without malignancy. Am J Respir Crit Care Med 2004; 170: 621-625. 